\title{
Microstructure and Formation Mechanisms of $\delta$-Hydrides in Variable Grain Size Zircaloy-4 Studied by Electron Backscatter Diffraction
}

\author{
Siyang Wang ${ }^{1}$, Finn Giuliani ${ }^{1}$, and T. Ben Britton ${ }^{1}$ \\ 1. Department of Materials, Imperial College London, SW7 2AZ
}

Zircaloy-4 is a critical material in current generation pressurized water reactors as nuclear fuel rod cladding. In service, the cladding can degrade due to the formation of hydrides as hydrogen ingresses during corrosion of the metal. These hydrides can significantly reduce the mechanical properties of the cladding [1] and represent a significant issue when considering the safety case of nuclear reactors. In this work, we explore the microstructural features and the formation mechanisms of $\delta$-hydrides in Zircaloy- 4 using electron backscatter diffraction (EBSD).

In the present work, we perform hydriding using electrolysis and thermal re-diffusion to create hydrides in polycrystalline Zircaloy-4. We have used two grain sizes of Zircaloy-4, one with an average grain size of $\sim 11 \mu \mathrm{m}$ and "blocky alpha" [2] with a grain size of $>200 \mu \mathrm{m}$. Hydriding of these samples was performed using an electrochemical cell with a platinum counter electrode and $1.5 \mathrm{wt} \%$ sulphuric acid with a current density of $2 \mathrm{kA} / \mathrm{m}^{2}$. After charging, the material was annealed at $400{ }^{\circ} \mathrm{C}$ (above the hydride solvus line) for 5 hours and then slow cooled at $0.5^{\circ} \mathrm{C} / \mathrm{min}$, which favors $\delta$-phase hydride formation [3,4]. The samples were metallographically polished and subsequently electropolished with $10 \mathrm{vol} \%$ perchloric acid in methanol at $-40{ }^{\circ} \mathrm{C}$ for $90 \mathrm{~s}$ under an applied voltage of $30 \mathrm{~V}$ to remove the surface stress layer and generate good quality EBSD patterns.

EBSD was performed on a FEI Quanta 650 scanning electron microscope (SEM) equipped with a Bruker eFlashHR (v2) EBSD camera using a beam accelerating voltage of $20 \mathrm{kV}$ and a probe current of $\sim 10 \mathrm{nA}$. Collection and analysis of the patterns was performed using ESPRIT 2.1. EBSD patterns were captured at $320 \times 240$ pixels, with an exposure time of $22 \mathrm{~ms}$.

We observe intergranular hydrides, intragranular hydrides, and twin boundary hydrides in the large grain material. Within the majority of these hydrides, we observe that the FCC $\delta$-hydride contains twins and that there are orientation relationships (OR) of $\{0001\} \alpha\|\{111\} \delta ;<11 \overline{2} 0>\alpha\|<110>\delta$ between the $\delta$ hydrides and the HCP $\alpha$-zirconium matrix.

We observe that the hydrides often form on $\mathrm{T} 1 \alpha-\mathrm{Zr}$ twin boundaries and the matrix-zirconium OR is maintained for the parent zirconium crystal which is the immediate neighbor of the hydride packet. Due to the nature of the twin, this means that there is an OR for the hydrides formed within the in the parent and twin which is a product of the T1 OR and the hydride OR.

For intragranular hydride internal hydride twins are found within the hydride packet. We also observe that the hydrides extend on the macroscopic $\{10 \overline{1} 7\}$ crystal plane, likely due to strain accommodation between the individual hydride platelets which seem to form on the $\{0001\}$ basal planes. The strain accommodation hints at sympathetic growth of the hydride as it extends into the twin.

In the fine grain material, sympathetic hydride growth is indicated due to the presence of significant 
hydride "stringers" which decorate grain boundaries and extend across multiple grains within the polycrystalline material. These stringers are observed to run perpendicular to the normal direction (ND) in this rolled Zircaloy-4 plate (which has a conventional split basal ND texture).

Careful observations of the hydride network as it extends through the polycrystalline network indicates that the hydride packets likely form sequentially and grow from each other. This is likely due to a local strain field effect (due to the significant misfit strain associated with the hydride tip) and the resulting change in local chemical potential distribution. The consequence of this effect is a significant, extensive and contiguous hydride film which has the potential to significantly influence the propagation of cracks while the cladding is in service.

The authors acknowledge funding from [5]. The preprint of this work can be found here: https://arxiv.org/abs/1811.12442

\section{References:}

[1] G. Bertolino, G. Meyer, J. Perez Ipiña, J. Nucl. Mater. 320 (2003) 272-279.

[2] V. Tong, B. Britton, Acta Mater. 129 (2017) 510-520.

[3] B. Nath, G.W. Lorimer, N. Ridley, J. Nucl. Mater. 58 (1975) 153-162.

[4] R. Birch, S. Wang, V. Tong, B. Britton, J. Nucl. Mater. 513 (2019) 221-225.

[5] TBB and SW acknowledge support from the HexMat programme grant (EP/K034332/1). TBB thanks the Royal Academy of Engineering for funding his Research Fellowship. The FEI Quanta SEM used was supported by the Shell AIMS UTC and is housed in the Harvey Flower EM suite at Imperial College London. We acknowledge Vivian Tong for useful discussions on this work.

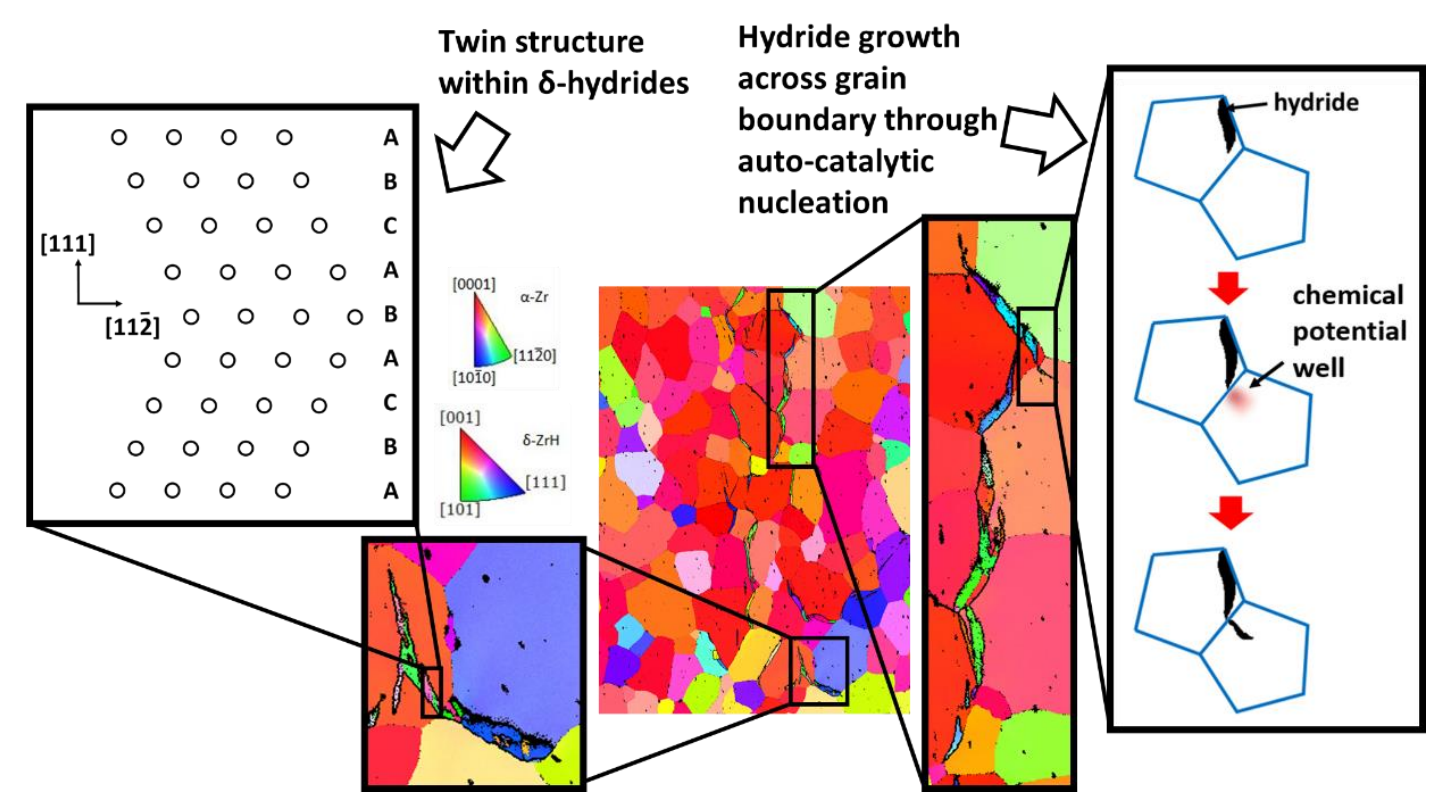

Figure 1. Structure, morphology and crystallography of $\delta$-hydrides revealed by EBSD indicating autocatalytic growth pattern across grain boundaries and twin formation due to misfit stress build-up. 